\title{
Shaping district diabetes services: a novel performance index scoring system to successfully negotiate with Clinical Commissioning Groups
}

\author{
EMMA RUSSELL-JONES'1, ROSELLE HERRING², KLAUS GREEN², VICTORIA HORDERN²
}

\begin{abstract}
Aims: To establish whether a recently reported performance index scoring system developed in two diverse populations (Medway and Guildford, UK) could be used to successfully negotiate with a Clinical Commissioning Group (CCG) to shape diabetes services and set priorities. Methods: We collated demographic details for the area's population with diabetes with diabetes scores from local primary care Quality and Outcomes Framework (QOF) records, together with exception reporting. Data from Hospital Episode Statistics and Dr Foster were used to record the Standardised Admission Rate (SAR) for a first/new referral to a secondary-care diabetes outpatient appointment or for emergency admission to hospital for diabetes. Hospital notes for patients from one GP practice were reviewed to clarify why patients were seen in secondary rather than primary care.

Results: The prevalence of diabetes was low (4\%) compared with figures from the Strategic Health Authority (SHA) (4.5\%) and nationally (5.6\%). Total diabetes QOF points achieved (99.3) were higher than for the SHA (97.8) and nationally (95.3); $61.1 \%$ achieved $\mathrm{HbA}_{1 \mathrm{c}}<7 \%$, which was much higher than the SHA $(57.2 \%)$ or national average (54.3\%). The exception-reporting rate $(9.8 \%)$ was lower than the SHA $(10.5 \%)$ or national average $(14 \%)$. The SAR was lower than the Surrey average for a first outpatient visit (87.8 vs. 128.9 ) or emergency admissions ( 85 vs. 106.7 ).

Conclusions: The performance index scoring system enabled an assessment of diabetes care that transcended primary and secondary care. A successful negotiation
\end{abstract}

Royal Sussex County Hospital, Brighton, UK

Royal Surrey County Hospital, Guildford, Surrey, UK

The Mill Surgery, Godalming, Surrey, UK

Address for correspondence: Emma Russell-Jones Cedar Centre, Royal Surrey County Hospital, Edgerton Road, Surrey, GU2 7XX, UK

Tel: +44 (0)1483 571122 Ext 4844

E-mail: emma.russell-jones@bsuh.nhs.uk

http://dx.doi.org/10.15277/bjdvd.2015.010

$\begin{array}{ll}\text { Abbreviations and acronyms } \\ \text { CCG } & \text { Clinical Commissioning Group } \\ \text { DSN } & \text { Diabetes Specialist Nurse } \\ \text { GP } & \text { General practitioner } \\ \text { LIT } & \text { Local Implementation Team } \\ \text { NHS } & \text { National Health Service } \\ \text { PCG } & \text { Primary Care Group } \\ \text { PCT } & \text { Primary Care Trust } \\ \text { QOF } & \text { Quality and Outcomes Framework } \\ \text { SAR } & \text { Standardised Admission Rate } \\ \text { SHA } & \text { Strategic Health Authority }\end{array}$

took place between the local diabetes stakeholders and the CCG with a plan for improving and developing the current model of care. The scoring system is applicable to any district in the UK and may be of interest to clinicians and commissioners.

Br J Diabetes Vasc Dis 2015;15:56-60

Key words: diabetes, performance index, quality, community, services

\section{Introduction}

The rising prevalence of diabetes worldwide and its resultant chronic disease burden and economic consequences is a cause of much concern. ${ }^{1,2}$ New CCGs are keen to encourage the primary care sector to take an increasing role in the provision of care for chronic conditions, and the majority of patients with type 2 diabetes are expected to be managed in primary care in future. Diabetes has been highlighted for special attention, as the projected health economic burden is enormous. ${ }^{3}$

Models of integrated care between the primary and secondary healthcare sectors have been described previously. For example, the Super Six model identified six areas of diabetes care which should be provided in the hospital setting, with all other care provided in a primary care setting. ${ }^{4}$ Alternatively, the Derby Integrated Care model described a social enterprise formed by clinicians interested in primary and secondary diabetes care. ${ }^{5}$ Concerns have been raised within the primary and secondary care sectors of the south-west Surrey district that a model of care would be imposed that would be inappropriate for the local patient population. A 
Figure 1. Current framework of support care for people with diabetes in south-west Surrey

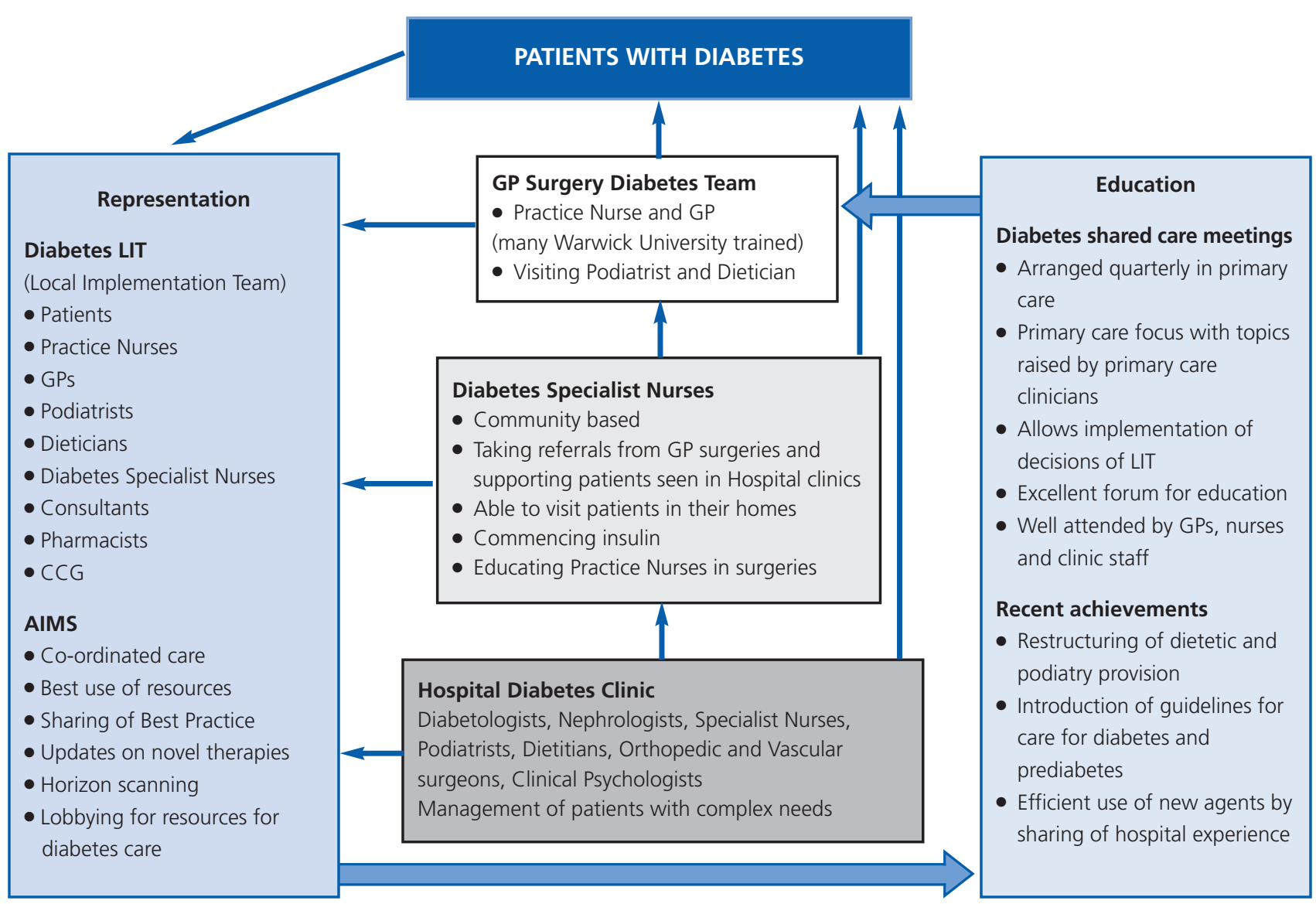

new performance scoring system was used by the LIT to provide evidence of current practice to the new CCG and aid negotiation of diabetes care. ${ }^{6}$ Here, we describe the design and utility of this new system.

\section{Methods}

\section{Diabetes care in south-west Surrey}

The diabetes integrated care model in south-west Surrey is tailored to local patient demographics. Figure 1 shows the current framework of support: there are clear lines of responsibility for the patient, with primary care playing the dominant role in a patient-focussed approach. Community DSNs employed by Virgin Care under a service specification agreement within a block contract for district nurses are central to this process; these nurses have an important role as educators to primary care practices and offer support with day-to-day patient management such as insulin initiation, treatment intensification and communication between primary and secondary care. Importantly, DSNs are based within the secondary care diabetes setting, which facilitates communication with secondary care professionals and their involvement at weekly diabetes team meetings, where difficult and complex patients are reviewed and referred to secondary care as necessary.

A number of initiatives have taken place to support the framework over the last 10 years. The PCT funded over 200 GPs and practice nurses to complete the Warwick Diploma in Diabetes. Pharmaceutical companies have provided financial support, but have no input into the educational content of quarterly shared care meetings, which update primary care professionals on recent advances in diabetes care and on local diabetes initiatives (these meetings have been described by the Department of Health as an example of best practice). In addition, the PCG and PCT have introduced a number of financial incentive schemes to enable rapid and safe discharge of patients from secondary care clinics. The LIT, chaired by a primary care professional, consists of lay representatives, DSNs, GPs and diabetologists, aims to coordinate care, optimise use of resources, share best practice, update on latest therapies and lobby for resources for diabetes care.

Developing a performance index for diabetes services in south-west Surrey

The LIT compiled a list of relevant primary care practices, designated the 'cluster'. The performance indicator system was 
derived using routinely collected and freely available data from local diabetes QOF records, ${ }^{7}$ Hospital Episode Statistics ${ }^{8}$ and Dr Foster. ${ }^{9}$ QOF provides a series of clinical performance indicators that are used to determine financial rewards for primary care practices when certain thresholds or targets are achieved. It is important to examine practice exemption rates as well as achievement rates for any of the named indicators when evaluating data from QOF.7 Dr Foster data comprise information gained for hospital trusts and primary care and are based around activity that was invoiced and paid for by PCTs. Many believe these data to be the most accurate measure of hospital activity. ${ }^{9}$ Information on the referral rate (SAR) for a first visit to diabetes secondary care, or to emergency care for diabetes, were obtained for the practices over a 12 month period.

One GP practice was selected for further investigation. This practice had a lower than expected referral rate to secondary care diabetes services and also a low emergency admission rate (although these referral rates were neither the highest nor lowest in the cluster of local practices). Notes were reviewed for all patients from that practice who were referred to a secondary care specialist diabetes clinic in order to establish the reason for referral and the nature of clinics attended.

\section{Results}

The prevalence of diabetes in south-west Surrey was low at 4\%, compared with the local SHA (Kent, Surrey \& Sussex) average of $4.5 \%$ and the national average of $5.6 \%$. Total diabetes QOF points achieved were higher (99.3\%) than the SHA average $(97.8 \%)$ or the national average $(95.3 \%)$. $\mathrm{HbA}_{1 \mathrm{c}}<7 \%$ was achieved by $61.1 \%$ of patients, which was higher than the SHA average $(57.2 \%)$ or the national average $(54.3 \%)$. There was a lower exception reporting rate of $9.8 \%$ compared with the average for the SHA (10.5\%) or nationally (14\%).

The average SAR for referral for first visit diabetes outpatients was low at $87.8 \%$ (percentage of observed/expected referrals; Surrey average $128.9 \%$ ) although there was variability between practices (Table 1). The SAR for an emergency diabetes-related admission was lower than expected (85\%), compared with the Surrey average of $106.7 \%$, once again with variation between practices (Table 1).

Table 1 SARs for first diabetes outpatient appointment in secondary care and for emergency admissions for diabetes for each practice. The expected SAR value was based on deprivation, prevalence and needs of practice.

\begin{tabular}{|c|c|c|c|c|c|c|}
\hline \multirow[b]{2}{*}{ Practice ID } & \multicolumn{3}{|c|}{ SAR for first diabetes outpatient appointment } & \multicolumn{3}{|c|}{ SAR for emergency admission for diabetes } \\
\hline & Observed & Expected & $\%$ & Observed & Expected & $\%$ \\
\hline H81044 & 24 & 49.26 & 48.7 & 3 & 3 & 100 \\
\hline H81043 & 69 & 134.75 & 51.2 & 3 & 5.63 & 53.3 \\
\hline H81031 & 57 & 107.56 & 53 & 3 & 3 & 100 \\
\hline H81077 & 45 & 83.71 & 53.8 & 3 & 3 & 100 \\
\hline H81085 & 74 & 115.68 & 64 & 3 & 3 & 100 \\
\hline H81029 & 97 & 145.78 & 66.5 & 3 & 6.33 & 47.4 \\
\hline H81052 & 125 & 184.07 & 67.9 & 3 & 7.82 & 38.3 \\
\hline H81021 & 123 & 178.42 & 68.9 & 6 & 7.57 & 79.3 \\
\hline H81113 & 72 & 101.53 & 70.9 & 3 & 3 & 100 \\
\hline H81062 & 158 & 220.53 & 71.6 & 3 & 9.26 & 32.4 \\
\hline H81132 & 22 & 27.95 & 78.7 & 3 & 3 & 100 \\
\hline H81053 & 69 & 84.86 & 81.3 & & & \\
\hline H81006 & 71 & 84.9 & 83.6 & 6 & 3 & 200 \\
\hline H81041 & 87 & 103.39 & 84.1 & 3 & 3 & 100 \\
\hline H81026 & 155 & 134 & 85.8 & 3 & 5.67 & 52.9 \\
\hline H81076 & 143 & 155.3 & 92.1 & 3 & 6.44 & 46.6 \\
\hline H81022 & 48 & 51.2 & 93.7 & 3 & 3 & 100 \\
\hline H81035 & 122 & 115.84 & 111.4 & 3 & 3 & 100 \\
\hline H81090 & 196 & 138.77 & 141.2 & 3 & 5.97 & 50.2 \\
\hline H81655 & 48 & 28.49 & 168.5 & 3 & 3 & 100 \\
\hline H81647 & 53 & 25.3 & 209.5 & 3 & 3 & 100 \\
\hline NHS Surrey & 17,769 & $13,787.96$ & 128.9 & 586 & 549.24 & 106.7 \\
\hline
\end{tabular}


Table 2 Reasons for attendance at a secondary care diabetes clinic for patients from one GP practice.

- Poor glycaemic control

- Severe microvascular complications (including blindness)

- Severe macrovascular complications

- $\quad$ Long duration with complications

- Coeliac and other autoimmune problems

- Unrecognised hypoglycaemia

- Malignancy

- Psychology input

- Gastroparesis

- Foot ulceration

- Severe obesity

- Cognitive impairment

- Alcohol excess

Fifty-two patients from the identified GP practice were seen in secondary care. The reasons for attendance at secondary care are shown in Table 2. Patients frequently seen in secondary care included antenatal and preconception care, patients requiring multidisciplinary foot care, joint renal diabetes support, patients with type 1 diabetes using insulin pump therapy and surgicallyinduced diabetes. The LIT deemed that the scope of attendance at the specialist clinics was appropriate, without unnecessary referrals.

\section{Discussion}

The performance index scoring system demonstrated that the prevalence of diabetes is relatively low in south-west Surrey. The performance of primary care diabetes services was good in terms of meeting agreed targets and process measures, referral to secondary care was low and the hospital admission rate was low, suggesting a cost-effective approach.

Health authorities are seeking to develop successful strategies to cope with the large financial burden of chronic diabetes care. ${ }^{1}$ The 2014-2015 NHS England CCG outcome indicator set a direction for the priorities of a new diabetes service, characterised by:

1. Supporting people in the management of their condition

2. Improving the functional ability of people who have received all of the nine care processes and structured education within 1 year of diagnosis

3. Reducing unplanned hospitalisation for problems related to diabetes including emergency admissions for diabetic ketoacidosis and amputations

4. Reducing the incidence of myocardial infarction and stage 5 renal failure in people with diabetes.

Any model must also resonate with the NHS priorities of:

- Patient focus and empowering

- Reduction in variation

- Improved outcomes

- Improved experience

- Financial sustainability

The UK has been the first country that has incentivised

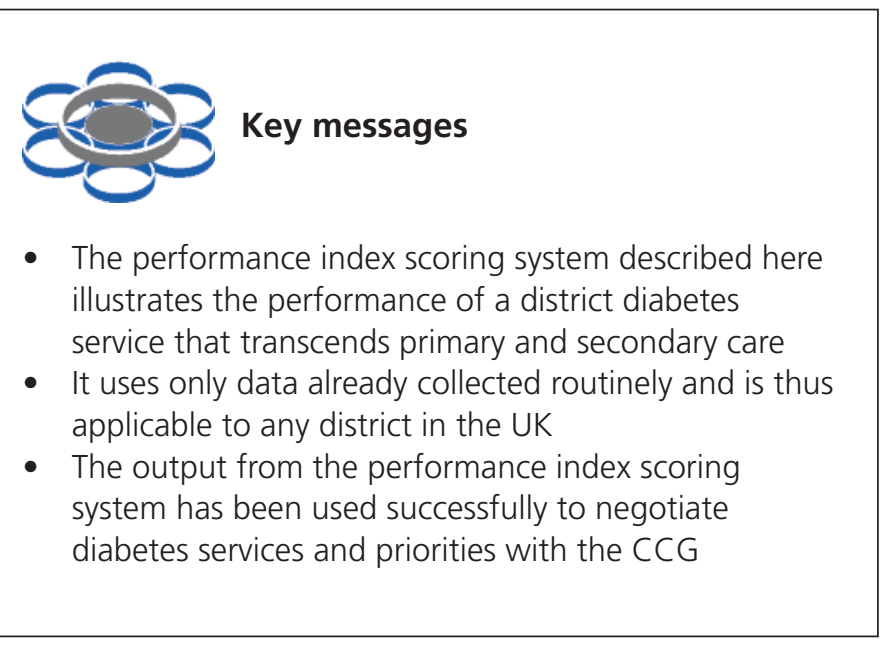

primary care physicians to improve diabetes care in the community with the implementation of the QOF indicators and local enhanced services.5,8 The LIT in south-west Surrey was able to use the performance index scoring system in diabetes to demonstrate the effectiveness of the district's diabetes services to new commissioners and managers in the CCG. It was also acknowledged that secondary care is needed to supplement primary care diabetes clinics and few patients were being seen unnecessarily in secondary care. CCG funding for the continuation of GP and practice nurse education programmes to increase the skill mix in primary care and continued access to the community DSNs were identified as key priorities to ensure the diabetes framework continues to work effectively. As a result of using this analytical approach, the CCG and commissioners were reassured by the performance of the current model of care and it was decided to evolve rather than redesign the service.

Community DSNs offer an open and equal service to all practices within the 'cluster' and are central to the model. DSNs accept referrals from GPs, practice nurses, district nurses, and inpatient specialist diabetes nurses, or patients can self-refer. Referrals are monitored and educational support is offered to healthcare professionals if there is a disproportionate amount of support directed to individual practices.

The limitations of the performance indicator index relate mainly to its simplicity and use of relatively crude measures. Follow-up activity has not been included and is probably not relevant as non-complex patients are managed in primary care. It is therefore expected that more complex chronic patients will be referred to secondary care and require longer follow-up with multidisciplinary input. However, a unique strength of the model is its applicability to any area of the UK as all data parameters are already being collected routinely. We have demonstrated that the performance index provides a number of important measures of service delivery. Individual districts could develop it further by weighting some of the individual parameters in terms of a pre-specified outcomes: for example, the avoidance of admission might be considered to be a more important outcome than the achievement of a reduced rate of outpatient referrals. 


\section{Conclusion}

Combining QOF data, Hospital Episode Statistics and Dr Foster and using a scoring system combined with a simple audit of hospital notes, it has been possible to illustrate the performance of a district diabetes service that transcends primary and secondary care. The data are very easy to access and freely available, and helped the CCG and commissioners to make informed decisions on service development. ${ }^{4}$

\section{Conflict of interest None. \\ Funding None.}

\section{References}

1. Danaei G, Finucane MM, Lu Y, et al. National, regional, and global trends in fasting plasma glucose and diabetes prevalence since 1980: systematic analysis of health examination surveys and epidemiological studies with 370 country-years and 2.7 million participants. Lancet 2011;378:31-40.
2. Guildford and Waverley Clinical Commissioning Group. Available at http://www.guildfordandwaverleyccg.nhs.uk (August 2014).

3. NHS England. Action for diabetes. Available at http://www.england nhs.uk/ourwork/qual-clin-lead/action-for-diabetes/ (August 2014)

4. Kar P. The Super Six model: Integrating diabetes care across Portsmouth and south-east Hampshire. Diabetes and Primary Care 2012;14:277-83.

5. Rea RD, Gregory S, Browne M, lqbal M, et al. Integrated diabetes care in Derby: New NHS organisations for new challenges. Pract Diab 2011:28:312-13. http://dx.doi.org/10.1002/pdi.1624

6. Russell-Jones EC, Gough A, Scobie IN. The Novel use of commonly captured data to assess a district's diabetes service that encompasses both primary and secondary care. QJM 2013;106:737-45. http://dx.doi.org/10.1093/qjmed/hct097

7. Quality Outcomes Framework indicators. Available at http://www.dhsspsni.gov.uk/qof-indicators.pdf (August 2014).

8. Hospital Episode Statistics. Available at http:// http://webarchive.nationalarchives.gov.uk/20130107105354/http://www.dh.gov.uk/en/Publicationsandstatistics/Statistics/HospitalEpisodeStatistics/index.htm (August 2014).

9. Dr Foster. Available at: http://www.drfosterhealth.co.uk/ (August 2014)

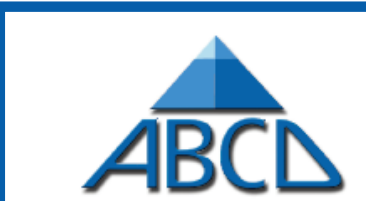

Association of British Clinical Diabetologists

\section{Insulin degludec (Tresiba) Nationwide Audit Now Launched!}

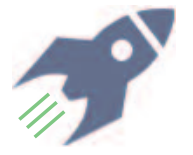

$A B C D$ has launched a nationwide audit of insulin degludec in the UK to assess real clinical efficacy and safety \& inform future practice and guidelines

Does your centre use insulin degludec?

\section{If yes, REGISTER YOUR CENTRE! by contacting degludec.audit@diabetologists.org.uk}

- you are invited to enter your patients' data into the bespoke online tool

- you are able to analyse your local data easily

- the data will be automatically added to the national data in anonymised form

- we can provide easy-to-complete paper proformas for use in clinic if preferred

Please remember: - the more data, the more complete our understanding of insulin degludec in real clinical practice will be

- all contributors will be listed in publications arising from data submission 